\title{
UPPER AND LOWER ESTIMATES ON THE RATE OF CONVERGENCE OF APPROXIMATIONS IN $H_{p}$
}

\author{
BY FRANK STENGER
}

Communicated by Walter Gautschi, June 1, 1977

Let $1<p \leqslant \infty$ and let $H_{p}(U)$ denote the family of all functions $f$ that are analytic in the unit disc $U$ and such that

$$
\|f\|_{p}=\lim _{r \rightarrow 1}\left(\frac{1}{2 \pi} \int_{0}^{2 \pi}\left|f\left(r e^{i \theta}\right)\right|^{p} d \theta\right)^{1 / p}<\infty .
$$

Let $\sigma_{n}$ be defined by

$$
\sigma_{n}=\inf _{w_{j} \in C, x_{j} \in U} \sup _{f \in H_{p}(U),\|f\|_{p}=1}\left|\int_{-1}^{1} f(x) d x-\sum_{j=1}^{n} w_{j} f\left(x_{j}\right)\right| .
$$

We announce the following result.

THEOREM 1. Given any $\epsilon>0$, there exists an integer $n(\epsilon) \geqslant 0$ such that whenever $n>n(\epsilon)$, then

$$
\exp \left[-\left(5^{1 / 2} \pi+\epsilon\right) n^{1 / 2}\right] \leqslant \sigma_{n} \leqslant \exp \left[-\left(\frac{\pi}{(2 q)^{1 / 2}}-\epsilon\right) n^{1 / 2}\right]
$$

where $q=p /(p-1)$.

Next, let $H_{p}^{*}(U)$ denote the family of all functions $g$ such that $f \in H_{p}(U)$, where $f(z)=g(z) /\left(1-z^{2}\right)$, and such that $H_{p}^{*}(U)$ is normed by $\|g\|_{p}^{*}=\|f\|_{p}$, where $\|f\|_{p}$ is defined as in (1). Let $g \in H_{p}^{*}(U)$, and let $\left\{T_{n}(g)\right\}$ be a linear approximation scheme defined by

$$
T_{n}(g)(z)=\sum_{j=1}^{n} g\left(x_{j}\right) \phi_{n, j}(z), x j \in U
$$

where $\phi_{n, j}$ is analytic in $U$ for each $n$ and $j$, and such that

$$
\left\|T_{n}(g)\right\|_{p}^{*} \leqslant C\|g\|_{p}^{*}
$$

where $C$ is independent of $n$. We then announce

AMS (MOS) subject classifications (1970). Primary 65D30, 65D15; Secondary 65D05, 65D15, 65D25.

1 Research supported by NRC Grants A-0201 and A-8240 of the University of British Columbia. 
THEOREM 2. Given any $\epsilon>0$, there exists an integer $n(\epsilon) \geqslant 0$, such that whenever $n>n(\epsilon)$, then

$$
\begin{aligned}
& \exp \left[-\left(5^{1 / 2} \pi+\epsilon\right) n^{1 / 2}\right] \\
& \leqslant \inf _{T_{n} g \in H_{p}^{*}(U),\|g\|_{p}^{*}=1} \sup _{-1<x<1}\left|g(x)-T_{n}(g)(x)\right| \\
& \quad \leqslant \exp \left[-\left(\frac{\pi}{2 q^{1 / 2}}-\epsilon\right) n^{1 / 2}\right] .
\end{aligned}
$$

Let us briefly mention some papers which are relevant to the present work. In 1964 Wilf [9] proved for the case $p=2$ that $\sigma_{n}=O\left((\log n / n)^{1 / 2}\right)$. In 1971 Haber [2] and Johnson and Riess [3] proved for $p=2$ that $\sigma_{n}=$ $O\left(n^{-1 / 2}\right)$. The authors of [2], [3] conjectured that their bound was the best bound possible. In 1973 [6] it was shown by the author that for $p=2, \sigma_{n}=$ $O\left(e^{-\pi n^{1 / 2}} / 2\right)$. In 1975 it was shown by Loeb and Werner [4] that for arbitrary $p>1, \sigma_{n} \leqslant 2^{1+2 / q} \exp \left[-(n / 2)^{1 / 2} /(2 q)\right]$.

The bounds of Theorem 1 are sharper than any others that have been obtained previously. While there is a gap in the constants of the upper and lower bounds, no one has previously obtained a lower bound. Moreover, no one has previously obtained upper or lower bounds of the type in Theorem 2, for approximation in $H_{p}^{*}(U)$.

The results of Theorems 1 and 2 may be extended to establishing the optimal $O\left(e^{-c n^{1 / 2}}\right)$ rate of convergence of quadrature and interpolation in other $H_{p}$ spaces, $p>1$. In what follows, we shall describe some of these. We shall also mention known methods of quadrature or interpolation in each case, which converge at the $O\left(e^{-a n^{1 / 2}}\right)$ rate. At this time it is not known whether or not $a=c$ for any of these methods.

(a) Let $0<d \leqslant \pi / 2$, let $D_{d}=\{z=x+i y$ : $|\arg [(1+z) /(1-z)]|<d\}$. (Note that $D_{\pi / 2}=U$ ) and let $H_{p}\left(D_{d}\right)$ denote the family of all functions $f$ that are analytic in $D_{d}$ such that

$$
\|f\|_{p}=\lim _{C \rightarrow \partial D_{d}, C \subset D_{d}} \inf _{C}\left(\int_{C}|f(z)|^{p}|d x|\right)^{1 / p}<\infty .
$$

The optimal rate of convergence of quadratures $(2)$ in $H_{p}\left(D_{d}\right)$ is $O\left(e^{-c n^{1 / 2}}\right)$, where

$$
(\pi d / q)^{1 / 2} \leqslant c \leqslant 5^{1 / 2} \pi+\epsilon, \quad \epsilon>0 \text { arbitrary. }
$$

The quadrature methods of Theorem 1.6(b) of [8] and Theorem 3.2 of [7] converge at the $O\left(\exp \left[-(\pi d / q)^{1 / 2} n^{1 / 2}\right]\right)$ rate.

(b) Let $H_{p}^{*}\left(D_{d}\right)$ denote the family of all functions $g$ such that $f \in H_{p}\left(D_{d}\right)$ 
where $f(z)=g(z) /\left(1-z^{2}\right)$ and where $H_{p}\left(D_{d}\right)$ is defined in (a) above. The optimal rate of convergence of interpolation (4) in $H_{p}^{*}\left(D_{d}\right)$ is $O\left(e^{-c n^{1 / 2}}\right)$, where

$$
[\pi d /(2 q)]^{1 / 2}-\epsilon \leqslant c \leqslant 5^{1 / 2} \pi+\epsilon, \quad \epsilon>0 \text { arbitrary. }
$$

The method [8]

$$
\left\{\begin{array}{l}
g(x) \cong \sum_{j=-N}^{N} g\left(x_{j}\right) S(j, h) \circ \log \left(\frac{1+x}{1-x}\right) \\
S(j, h)(x)=\frac{\sin [\pi(x-j h) / h]}{[\pi(x-j h) / h]} \\
h=(\pi d q / N)^{1 / 2}, x_{j}=\tanh (j h / 2)
\end{array}\right.
$$

converges at the $O\left(\exp \left\{-\left([\pi d /(2 q)]^{1 / 2}-\epsilon\right) n^{1 / 2}\right\}\right)$ rate, where $n=2 N+1$, and $\epsilon>0$ is arbitrary.

(c) Let $D_{d}=\{z=x+i y:|y|<d\}$, and let $H_{p}\left(D_{d}\right)$ denote the family of all functions $f$ that are analytic in $D_{d}$ such that

$$
N(f, y)=\left(\int_{R}\left\{|f(x+i y)|^{p}+|f(x-i y)|^{p}\right\} \cosh ^{2 p / q}(x / 2) d x\right)^{1 / p}<\infty
$$

$y<d$, and such that $\|f\|_{p}=N\left(f, d^{-}\right)<\infty$.

(i) The optimal rate of convergence of $n$-point quadratures

$$
\int_{R} f(x) d x \cong \sum_{j=1}^{n} w_{j} f\left(x_{j}\right)
$$

in $H_{p}\left(D_{d}\right)$ is $O\left(e^{-c n^{1 / 2}}\right)$, where $c$ is subject to (8). The trapezoidal rule,

$$
\int_{R} f(x) d x \cong h \sum_{j=-N}^{N} f(j h), \quad h=(2 \pi d q / N)^{1 / 2},
$$

converges at the $\exp \left[-(\pi d / q)^{1 / 2} n^{1 / 2}\right]$ rate [8], where $n=2 N+1$.

(ii) The optimal rate of interpolation of $f \in H_{p}\left(D_{d}\right)$ on $R$ is $O\left(e^{-c n^{1 / 2}}\right)$, where $c$ is subject to (9). Interpolation via the Whittaker cardinal function,

$$
f(x) \cong \sum_{j=-N}^{N} f(j h) S(j, h)(x) \quad\left(h=(\pi d q / N)^{1 / 2}\right)
$$

converges at the $\left.O\left(\exp \left\{-([\pi d / 2 q)]^{1 / 2}-\epsilon\right) n^{1 / 2}\right\}\right)$ rate [8], where $n=2 N+1$, and $\epsilon>0$ is arbitrary.

\section{REFERENCES}

1. A. A. Goncar, On the rapidity of rational approximation of continuous functions with characteristic singularities, Math. USSR-Sb. 2 (1967), 561-568. 
2. S. Haber, The error in the numerical integration of analytic functions, Quart. Appl. Math. 29 (1971), $411-420$.

3. L. W. Johnson and R. D. Riess, Minimal quadratures for functions of low order continuity, Math. Comp. 25 (1971), 831-835.

4. H. L. Loeb and $\mathrm{H}$. Werner, Optimal numerical quadratures in $H_{p}$ spaces, Math. Z. 138 (1974), $111-117$.

5. L. Lundin and F. Stenger, Cardinal-type approximations of a function and its derivatives (submitted).

6. F. Stenger, Integration formulas based on the trapezoidal formula, J. Inst. Math. Appl. 12 (1973), 103-114.

7. - An analytic function which is an approximate characteristic function, SIAM J. Numer. Anal. 12 (1975), 239-254.

8. - Approximations via Whittaker's cardinal function, J. Approximation Theory 17 (1976), 222-240.

9. H. Wilf, Exactness conditions in numerical quadrature, Numer. Math. 6 (1964), $315-319$.

DEPARTMENT OF MATHEMATICS, UNIVERSITY OF BRITISH COLUMBIA, VANCOUVER, V6T1W5 BRITISH COLUMBIA, CANADA 